\title{
Solar Wind Sputtering of Wollastonite as a Lunar Analogue Material - Comparisons between Experiments and Simulation
}

Paul S. Szabo ${ }^{\text {a, }}$, Rimpei Chiba ${ }^{\text {a, }}$, Herbert Biber ${ }^{a}$, Reinhard Stadlmayr ${ }^{\mathrm{a}}$, Bernhard M. Berger ${ }^{\mathrm{a}}$, Daniel Mayer $^{\mathrm{a}}$, Andreas Mutzke ${ }^{\mathrm{b}}$, Michael Doppler ${ }^{\mathrm{c}}$, Markus Sauer ${ }^{\mathrm{d}}$, Julia Appenroth ${ }^{\mathrm{c}}$, Jürgen Fleig ${ }^{\mathrm{c}}$, Annette

Foelske-Schmitz $^{\mathrm{d}}$, Herbert Hutter ${ }^{\mathrm{c}}$, Klaus Mezger ${ }^{\mathrm{e}}$, Helmut Lammer ${ }^{\mathrm{f}}$, André Galli ${ }^{\mathrm{g}}$, Peter Wurz ${ }^{\mathrm{g}}$, and Friedrich Aumayr ${ }^{*}$

${ }^{a}$ Institute of Applied Physics, TU Wien, Wiedner Hauptstraße 8-10, 1040 Vienna, Austria

${ }^{\mathrm{b}}$ Max-Planck-Institut für Plasmaphysik, Wendelsteinstraße 1, 17491 Greifswald, Germany

${ }^{\mathrm{c}}$ Institute of Chemical Technologies and Analytics, TU Wien, Getreidemarkt 9, 1060 Vienna, Austria

d Analytical Instrumentation Center, TU Wien, Getreidemarkt 9, 1060 Vienna, Austria

${ }^{\mathrm{e}}$ Institute of Geological Sciences, University of Bern, Baltzerstraße 1+3, 3012 Bern, Switzerland

${ }^{\mathrm{f}}$ Space Research Institute (IWF), Austrian Academy of Sciences, Schmiedlstr. 6, 8042 Graz, Austria

${ }^{\mathrm{g}}$ Physics Institute, University of Bern, Sidlerstrasse 5, 3012 Bern, Switzerland

Keywords: Sputtering, Erosion, Quartz Crystal Microbalance, Solar Wind, Space Weathering, Exosphere

*Corresponding authors : szabo@iap.tuwien.ac.at, aumayr@iap.tuwien.ac.at

" present address: Tokyo Institute of Technology, Meguro-ku, Tokyo, 152-8550 Japan 


\begin{abstract}
The sputtering of wollastonite $\left(\mathrm{CaSiO}_{3}\right)$ by solar wind-relevant ions has been investigated experimentally and the results are compared to the binary collision approximation (BCA) codes SDTrimSP and SRIM-2013. Absolute sputtering yields are presented for Ar projectiles as a function of ion impact energy, charge state and impact angle as well as for solar wind $\mathrm{H}$ projectiles as a function of impact angle. Erosion of wollastonite by singly charged Ar ions is dominated by kinetic sputtering. The absolute magnitude of the sputtering yield and its dependence on the projectile impact angle can be well described by SDTrimSP as long as the actual sample composition is used in the simulation. SRIM-2013 largely overestimates the yield especially at glancing impact angles. For higher Ar charge states, the measured yield is strongly enhanced due to potential sputtering. Sputtering yields under solar wind-relevant $\mathrm{H}^{+}$bombardment are smaller by two orders of magnitude compared to Ar. Our experimental yields also show a less pronounced angular dependence than predicted by both BCA programs, probably due to $\mathrm{H}$ implantation in the sample. Based on our experimental findings and extrapolations to other solar wind ions by using SDTrimSP we present a model for the complete solar wind sputtering of a flat wollastonite surface as a function of projectile ion impact angle, which predicts a sputtering yield of 1.29 atomic mass units per solar wind ion for normal impact. We find that mostly He and some heavier ions increase the sputtering yield by more than a factor of two as compared to $\mathrm{H}^{+}$bombardment only.
\end{abstract}




\section{Introduction}

Space weathering represents one of the key topics in planetary science, describing the erosion and transformation of surfaces of rocky bodies in the solar system (Grün et al., 2011; Hapke, 1986; Pieters and Noble, 2016). The combination of external influences such as meteorite impacts, electromagnetic radiation and bombardment of ions will lead e.g. to changes of the optical properties of the surface and to evaporation or ejection of surface particles (Hapke, 2001). As a result of this emission, a tenuous exosphere is formed around the body that can be investigated (e.g. by spectroscopic techniques) to allow conclusions about the surface composition without the necessity of landing a spacecraft on the planet, moon or asteroid. Such missions have been performed in the past, for example the MESSENGER mission to Mercury by NASA (Vervack et al., 2010), and are planned in the future, such as the BepiColombo mission of ESA (Orsini et al., 2010).

For the release of refractory species, impact vaporization and ion sputtering are dominant (Wurz et al., 2010). On the Moon or on Mercury, sputtering is mainly caused by solar wind ions, which are mostly made up of protons $(\sim 93 \%)$ and He ions $(\sim 7 \%)$ (e.g. Wurz, 2005 and references therein) with a small part of heavier, multiply charged ions. However, due to the comparably low sputtering yields of protons, the heavy ions, especially He, must not be neglected for solar wind sputtering (Barghouty et al., 2011; Hijazi et al., 2017; Hijazi et al., 2014). The upper surface of planets and moons mostly consists of O-rich minerals, a fact that leads to a combination of different sputtering effects. Since these minerals are insulating materials, the importance of sputtering by heavier ions is even more increased by potential sputtering effects (Aumayr and Winter, 2004; Kracher et al., 2003). In addition, preferential sputtering leads to a change in the surface composition and consequently also affects the erosion rate. For example, preferential sputtering of $\mathrm{O}$ atoms causes a metallization of the surface (Hayderer et al., 2001; Loeffler et al., 2009), which can be connected to observed optical changes due to space weathering (Hapke, 2001).

Taking into account all occurring release processes, simulations can be performed to calculate exosphere densities (Pfleger et al., 2015; Wurz et al., 2007). Currently very little relevant experimental data for solar wind sputtering is available and exosphere models 
heavily rely on SRIM simulations (Ziegler et al., 2010) for sputtering yield inputs.

Therefore, validating SRIM simulations for composite minerals is a very important topic. For our experimental investigations of solar wind relevant sputtering, wollastonite $\left(\mathrm{CaSiO}_{3}\right)$ was chosen as an analogue material for the Moon's surface. Wollastonite is similar to minerals of the pyroxene group, which have been found in lunar regolith. In this work both experimental and theoretical investigations of the sputtering of wollastonite samples were performed. Sputtering yields were measured under both Ar and $\mathrm{H}$ irradiation and the experimental results were compared to SDTrimSP (Mutzke et al., 2011) and SRIM calculations (Ziegler et al., 2010). SRIM is almost exclusively used in the space weathering community, but SDTrimSP has been found to provide better results for sputtering simulations (Hofsäss et al., 2014). Additionally, the dynamic mode of SDTrimSP allows calculating changes in the target and its 2D-version SDTrimSP-2D (Mutzke et al., 2013) includes the surface roughness of the sample in its calculations. Furthermore, in this paper we investigate how effects such as potential sputtering and projectile implantation that cannot be described with conventional static simulations affect the sputtering yields of wollastonite.

The QCM-method that was used for the measurement of mass changes will be presented in Section 2 along with the analysis methods that were used to investigate the wollastonite samples and the simulation software that provided theoretical results for the sputtering yields. Afterwards, Section 3 shows the results of both experiments and simulations and discusses the differences found therein. Finally, the knowledge gained from the sputtering experiments is applied in Section 4 to model the complete solar wind sputtering yields of wollastonite under different angles of incidence. 


\section{Methods}

\subsection{Experimental Setup}

The device used for the sputtering experiments is a Quartz Crystal Microbalance (QCM), which allows performing mass change measurements in situ and in real time with very high precision (Hayderer et al., 1999). A plano-convex, stress compensated-cut quartz crystal from KVG Quartz Crystal Technology GmbH was used, where a thin film of wollastonite was deposited on top of the quartz's gold electrode (see Section 2.2 for a more detailed description of this process). During a sputtering experiment, this thin film is irradiated with an ion beam while the change of the quartz's resonance frequency is continuously measured. The QCM oscillates in a driven thickness shear mode at a base resonance frequency of about $6 \mathrm{MHz}$. According to Sauerbrey (Sauerbrey, 1959), if the mass change across the quartz's surface is uniform, the relative change of the resonance frequency $\Delta f_{Q} / f_{Q}$ is proportional to the relative thickness change $\Delta d_{Q} / d_{Q}$ and therefore to the relative mass change $\Delta m_{Q} / m_{Q}$ of the quartz:

$$
\frac{\Delta f_{Q}}{f_{Q}}=-\frac{\Delta d_{Q}}{d_{Q}}=-\frac{\Delta m_{Q}}{m_{Q}}
$$

Obtaining the mass change $\Delta m_{Q}$ of the quartz and measuring the ion beam current density $j$ then allows calculating the sputtering yield $y$, which describes the target mass change per incident ion in atomic mass units:

$$
y[\mathrm{amu} / \mathrm{ion}]=\frac{\rho_{Q} l_{Q}}{f_{Q} m_{n}} \cdot \frac{\Delta f_{Q}}{\Delta t} \cdot \frac{e_{0} q}{j}
$$

The first term includes the density of the quartz $\rho_{Q}$, its thickness $l_{Q}$, its resonance frequency $f_{Q}$ (which is assumed to be constant due to small changes $\Delta f_{Q}$ ) and the atomic mass unit $m_{n}=1.660 \cdot 10^{-27} \mathrm{~kg}$. This has to be multiplied with the frequency change over time and the inverse of the ion particle flux, which includes the electron charge $e_{0}$, the ions' charge state $q$ and the ion beam current density $j$. 
The QCM technique represents a very precise method for measuring sputtering yields (frequency changes as low as $1 \mathrm{mHz} / \mathrm{min}$ and mass changes as low as $40 \mathrm{pg} / \mathrm{s}$ can be obtained). However, only the absolute mass change can be measured and no information about the elemental composition of the sputtered material is available. For a more detailed description of the electronic setup of the QCM and the data evaluation see (Hayderer et al., 1999) and (Golczewski et al., 2009).

The QCM irradiation experiments were performed at the ion beam setup AUGUSTIN at the Institute of Applied Physics (IAP) at TU Wien which has been described in (Dobes et al., 2012). The QCM is mounted on a specifically designed sample holder inside an ultrahigh vacuum chamber, which is operated at a base pressure of about $10^{-10} \mathrm{mbar}$. Ions are produced in a $14.5 \mathrm{GHz}$ electron cyclotron resonance (ECR) ion source (Galutschek et al., 2007) and after extraction, the ion beam is mass-separated using a sector magnet. Using electrostatic lenses and deflection plates, the beam is focused and guided into the sample chamber. There scanning plates are used to create a uniform beam profile across the QCM target, which is necessary for the Sauerbrey equation (1) to hold. Additionally, an aperture that is mounted directly in front of the sample is used to control the shape of the scanned ion beam allowing circular shapes with diameters between 2 and $7 \mathrm{~mm}$ (Berger et al., 2017). Typical Ar fluxes of $10^{12}$ atoms $\mathrm{cm}^{-2} \mathrm{~s}^{-1}$ and $\mathrm{H}$ fluxes of $10^{13}$ atoms $\mathrm{cm}^{-2} \mathrm{~s}^{-1}$ were achieved for uniform scanning of the targets. The beam current density $j$ is measured before and after each experiment by a small Faraday cup with a precisely known opening and secondary electron suppression included in the QCM sample holder. During the irradiation itself, no information about the absolute value of the current is available, but its stability can be monitored by measuring the current on the aperture in the sample chamber. The sample holder is mounted on a rotatable manipulator to allow experiments with different angles of incidence. No heating of the QCM was performed during the measurements presented in this work and the sputtering yields were obtained for wollastonite target films at room temperature.

\subsection{Sample Preparation and Analysis}

Sample preparation started from a natural mineral sample of wollastonite. After polishing the rock with a wet-grinding machine it was heated for three days at a temperature of 
$320^{\circ} \mathrm{C}$ and a pressure of $10^{-2}$ mbar to remove all water from the stone. Then the sample was transferred to a pulsed laser deposition (PLD) apparatus, where thin films of the material were deposited on a few quartz crystals as well as on several Si single crystal plates for later analysis. The deposition of wollastonite films of several hundreds of nanometers thickness was performed with a $\mathrm{KrF}$ excimer laser (wavelength: $248 \mathrm{~nm}$, pulse frequency: $5 \mathrm{~Hz}$, pulse energy: $400 \mathrm{~mJ} /$ pulse) and a continuous sample temperature of $250{ }^{\circ} \mathrm{C}$ in the presence of an ambient oxygen gas at a pressure of $4 \cdot 10^{-2}$ mbar. The duration of the deposition process was varied between 30 minutes and 2 hours.
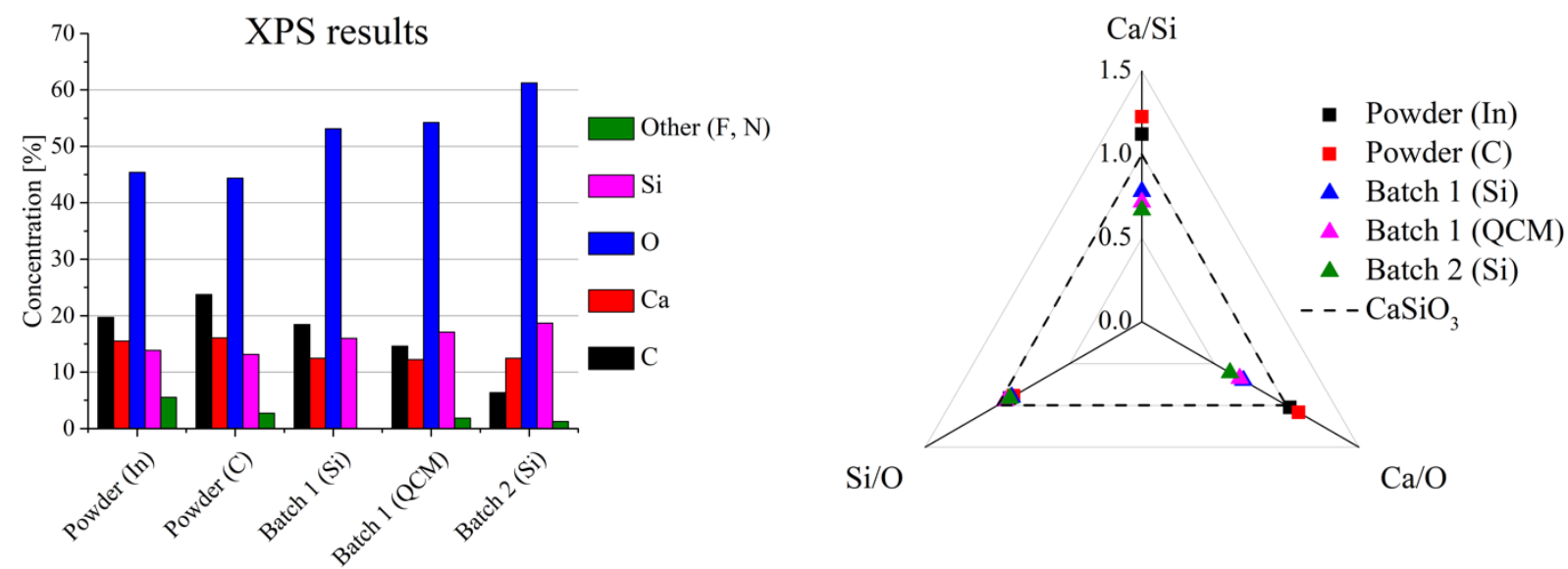

Fig. 1 Left: results of the XPS analysis for the different wollastonite samples. Right: relative abundances of the wollastonite constituents $\mathrm{Ca}, \mathrm{Si}$ and $\mathrm{O}$ for the same samples.

\begin{tabular}{|c|cccc|ccc|}
\hline Sample & C [\%] & Ca [\%] & O [\%] & Si [\%] & Ca/Si & $\mathbf{C a} / \mathbf{O}$ & Si/O \\
\hline Powder (In) & 19.69 & 15.52 & 45.42 & 13.85 & 1.12 & 0.34 & 0.31 \\
Powder (C) & 23.75 & 16.05 & 44.36 & 13.13 & 1.23 & 0.36 & 0.30 \\
\hline Batch 1 (Si) & 18.45 & 12.44 & 53.13 & 15.98 & 0.78 & 0.23 & 0.30 \\
Batch 1 (QCM) & 14.58 & 12.24 & 54.22 & 17.11 & 0.72 & 0.23 & 0.32 \\
\hline Batch 2 (Si) & 6.36 & 12.46 & 61.26 & 18.67 & 0.67 & 0.20 & 0.30 \\
\hline CaSiO $_{3}$ & - & 20.00 & 60.00 & 20.00 & 1.00 & 0.33 & 0.33 \\
\hline
\end{tabular}

Table 1 Summary of the results of the XPS analyses, and respective values for the nominal wollastonite composition $\mathrm{CaSiO}_{3}$ are included for comparison.

X-Ray Photoelectron Spectroscopy (XPS) was used to investigate the elemental composition of the samples and to compare the deposited films to the original rock. Both the original material (grinded to powder) as well as the PLD films were analyzed, with 
the results being presented in Fig. 1 and Table 1. For the XPS analysis of the original sample a part of the wollastonite rock was grinded into powder with a mortar and the powder was attached to In and C foils, as otherwise charging up would have disturbed a correct interpretation of the XPS results. For the PLD-produced thin films two Si plates from different batches as well as an actual quartz crystal used in the sputtering experiments were investigated by XPS. The left image in Fig. 1 shows a comparison of the different samples' compositions and the right image gives an overview of the abundance ratios for the wollastonite elements $\mathrm{Ca} / \mathrm{Si}, \mathrm{Ca} / \mathrm{O}$ and $\mathrm{Si} / \mathrm{O}$. Generally, the elemental composition of all samples (original as well as deposited) is very similar, indicating a feasible and reproducible deposition process via PLD. Only the relative Ca content has been found to be somewhat smaller in the thin film samples than in the original material. Variations in $\mathrm{C}$ and $\mathrm{O}$ probably occurred due to the immediate exposure to atmospheric conditions as deposition, analysis and later also irradiation were performed in different vacuum environments.
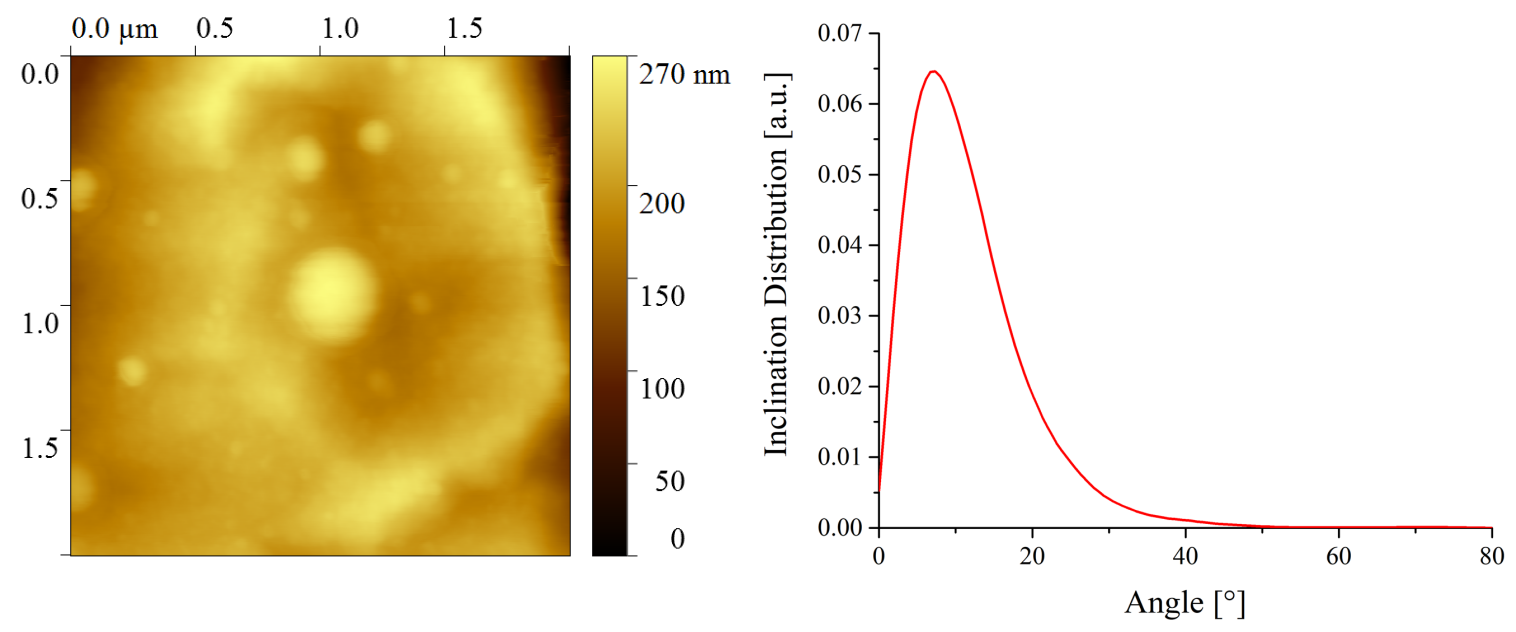

Fig. 2 Left: AFM image of a wollastonite film on a quartz crystal. Right: distribution of the surface's inclination angles. 


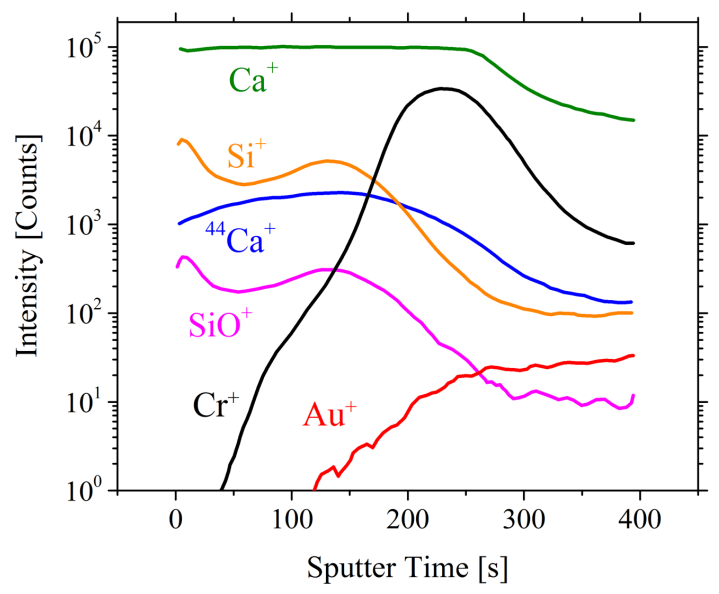

Fig. 3 TOF-SIMS analysis of a coated quartz crystal.

The surface topography of the deposited films on a quartz was probed with an Atomic Force Microscope (AFM), with a typical image of a wollastonite-coated quartz crystal sample being shown on the left in Fig. 2. Small circular features are observed with diameters between $100 \mathrm{~nm}$ and $500 \mathrm{~nm}$ across a generally much smoother base surface, where an RMS roughness value of $3.2 \mathrm{~nm}$ could be calculated from these AFM images. The distribution of inclination angles is shown on the right in Fig. 2, where most of the angles are found to be between 0 and 20 degrees, indicating a rather smooth surface. The circular features are not unusual in PLD-produced films and could consist of clusters or small chunks of material deposited together with the evaporated atoms. The smooth background and the circular features are both made up of deposited wollastonite, as the complete lack of any Au traces in the QCM's XPS analysis shows. To further confirm this, one of the quartzes was investigated with Time-of-Flight Secondary Ion Mass Spectroscopy (TOF-SIMS) under constant sputtering to achieve depth-dependent information about the film's composition. The TOF-SIMS results are shown in Fig. 3 where the yields for different secondary ions are plotted versus sputtering time. Although an absolute quantification is not possible for these SIMS measurements, they clearly show that Au ions from the quartz's top electrode can only be registered after prolonged sputtering. This was confirmed across multiple spots of investigation so that we can conclude that the whole surface of the quartz is covered by a closed layer of material, with an elemental composition closely resembling that of wollastonite rock. 


\subsection{Sputtering Simulations}

Sputtering of wollastonite was not only investigated experimentally, but also analyzed by using two binary collision approximation (BCA) programs, which are commonly applied to theoretically derive ion sputtering yields: SDTrimSP (Mutzke et al., 2011) and the TRIM simulation in the SRIM-2013 package (Ziegler et al., 2010). In Section 3 experimental and simulated results will be compared and the suitability of SDTrimSP and SRIM-2013 to simulate sputtering processes for materials such as wollastonite will be discussed.

As an input for both simulation programs the elemental composition of the sample was taken from the XPS analysis presented in Section 2.2. For SDTrimSP, the surface binding energies for the composite material were calculated by averaging the elemental surface binding energies provided by SDTrimSP (controlled with the parameter isbv=3 in the "tri.inp" input file (see Mutzke et al., 2011 for detailed information). Otherwise only standard parameters for both programs were used to investigate how well these codes are able to describe the sputtering of wollastonite. This also holds for the target density, which was calculated by the programs to be $1.587 \mathrm{~g} / \mathrm{mm}^{3}$ and is thus lower than the nominal crystalline wollastonite density of $2.86 \mathrm{~g} / \mathrm{mm}^{3}$. However, since XRD analysis of the deposited films showed no visible wollastonite peaks, an amorphous target with the standard density of the BCA simulations was assumed.

Since neither of the used programs takes the sample's surface structure into account, results are representative for a flat surface. The 2D version of SDTrimSP (SDTrimSP-2D) would in principle allow including the roughness of the target (Mutzke et al., 2013). However due to the target's low RMS roughness value of $3.2 \mathrm{~nm}$ and the occurrence of largely smooth areas on the target (see Fig. 2), a flat sample was adequate for the simulations. The assumption of a flat surface is backed by recent results of von Toussaint et al., who found a roughness independent sputtering yield for sample-RMS values of up to about $3 \mathrm{~nm}$ (von Toussaint et al., 2017). Realistically, flat surfaces are not representative for bodies exposed to the solar wind. However, we will present an investigation of the sputtering yield as a function of ion impact angle and rough surfaces can be - as a first approximation - described by a distribution of local impact angles (Küstner et al., 1998). 
In a more complex description, additional effects such as shadowing or redeposition of sputtered material have to be taken into account (Küstner et al., 1999; von Toussaint et al., 2017). Here the enhanced version SDTrimSP-2D (Mutzke et al., 2013) or the 3D version, which is currently under development, will have to be used for more precise sputtering calculations (von Toussaint et al., 2017). Nevertheless, the quality of simulations of rough targets are always based on a correct description of the sputtering of a flat target, so this case has to be validated first.

Angular and energy dependent sputtering yields of wollastonite were calculated with both SDTrimSP and SRIM-2013. The respective results are presented in Section 3 and compared to the experimentally derived values. 


\section{Results and Discussion}

\subsection{Angular Dependence under Ar Ion Irradiation}

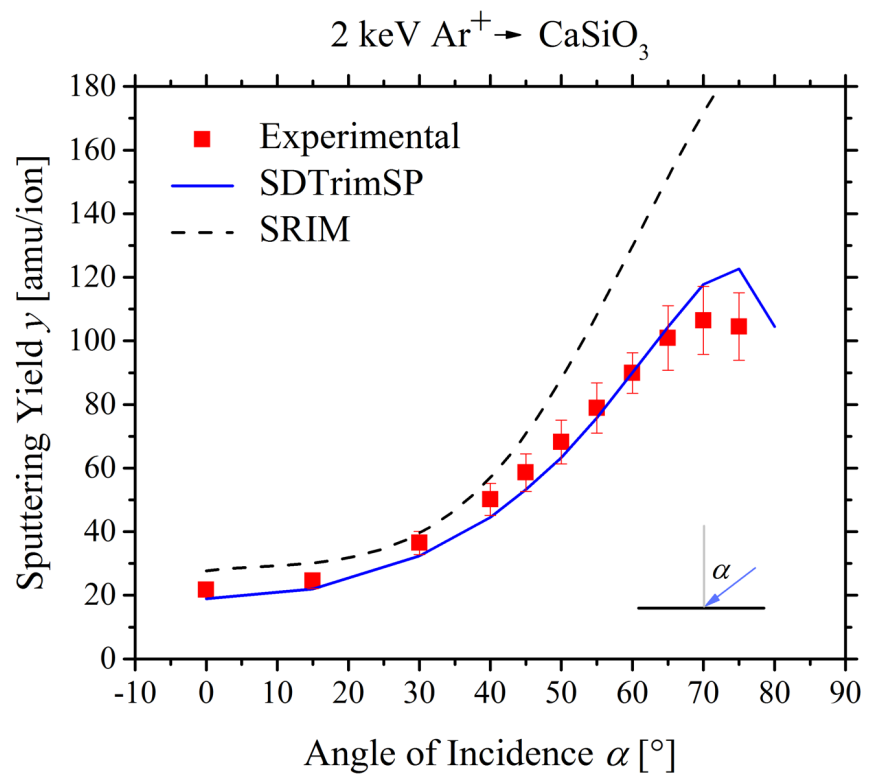

Fig. 4 Angular dependence of wollastonite sputtering under $2 \mathrm{keV} \mathrm{Ar}^{+}$irradiation.

The angular dependence of the wollastonite sputtering yield was investigated for the case of irradiation by $2 \mathrm{keV} \mathrm{Ar}{ }^{+}$ions. Ar is only a minor constituent of the solar wind, with its relative concentration compared to $\mathrm{H}$ being about $4 \cdot 10^{-6}$ (Barghouty et al., 2011).

However, it is well-suited for comparisons between experiment and simulation due to its high sputtering yield. Fig. 4 presents the results from the bombardment of a QCM (batch 1, red squares in the graph) along with simulated results from SDTrimSP (blue line) and SRIM-2013 (black dashed line). There the sputtering yield in amu/ion is plotted versus the ions' incidence angle with respect to the surface normal and thus 0 degree representing normal incidence. In the experiment, the wollastonite sputtering yield increases dramatically with increasing impact angle starting at about $20 \mathrm{amu} /$ ion for normal incidence and increasing to about $100 \mathrm{amu} / \mathrm{ion}$ at an incidence angle of 70 degrees. This behavior is very well reproduced by the SDTrimSP simulation, which only shows minor deviations for shallow angles of incidence. The SRIM simulations give slightly higher values of $y$ for angles below 45 degrees, but predict a much steeper increase for more gracing incidence, with deviations well outside our experimental error 
bars. Here SDTrimSP seems to be much better suited to describe the sputtering of wollastonite by $\mathrm{Ar}^{+}$ions than SRIM.

The investigation of wollastonite sputtering under $\mathrm{Ar}^{+}$bombardment can be considered as a model system for kinetic sputtering. As a noble gas, $\mathrm{Ar}^{+}$will not react chemically with the target material, there is no chemical sputtering (Hopf et al., 2003), and its potential energy of $15 \mathrm{eV}$ (DREEBIT, 2018) is too low to cause a noticeable potential sputtering effect (Aumayr and Winter, 2004). Under such circumstances, SDTrimSP obviously is able to predict the sputtering yield of a flat target extremely well (maximal error of about $10 \%$ ). However, such a good agreement is only reached if the actual sample composition is used in the simulation. For example, disregarding the XPS results and assuming the nominal $\mathrm{CaSiO}_{3}$ composition (last line in Table 1) would lead to an over-estimation of the sputtering yield by a factor of 1.5 .

Looking at differences in the standard input parameters for SDTrimSP and SRIM, not many discrepancies can be found. Target densities for the simulated amorphous material are $1.587 \mathrm{~g} / \mathrm{mm}^{3}$ in SDTrimSP and $1.736 \mathrm{~g} / \mathrm{mm}^{3}$ in SRIM. SDTrimSP uses a lower O displacement energy of $5 \mathrm{eV}$ and a higher surface binding energy of $2.58 \mathrm{eV}$, compared to $28 \mathrm{eV}$ and $2 \mathrm{eV}$ in SRIM respectively. Otherwise all other values show only minor differences. Even with similar input values, the SRIM results hardly change and exceed the SDTrimSP values as well as the experimental data. A closer look at an incidence angle of 60 degrees shows that this is mostly caused by an $\mathrm{O}$ sputtering yield of 3.53 (compared to 2.8 for SDTrimSP) and a Ca yield of 1.23 (compared to 0.57 for SDTrimSP), while the SRIM yield of Si is only slightly higher and the C yield is even lower than in SDTrimSP.

It can therefore be concluded that differences between SDTrimSP and SRIM-2013 cannot solely be explained by different input parameters, but the programs' approaches for simulating collisions and stopping of the projectiles must be responsible for the significant differences in the calculated sputtering yields. 


\subsection{Kinetic and Potential Energy Dependence under Ar Ion Irradiation}

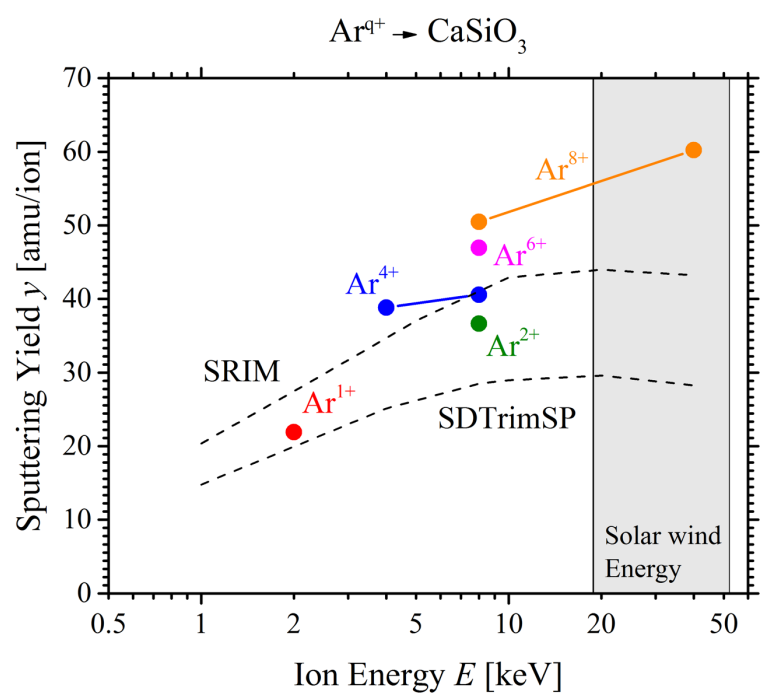

Fig. 5 Measured sputtering yield of wollastonite under normal incidence for Ar ion projectiles of different energies and charge states (colored dots) in comparison to SDTrimSP and SRIM results.

The wollastonite sputtering yield was also investigated under normal incidence for different Ar charge states and ion impact energies. Experimental results in Fig. 5 show that the sputtering yield increases both with increasing kinetic energy as well as increasing projectile charge states. The latter can be attributed to potential sputtering (Aumayr and Winter, 2004), a process active particularly for insulating materials. BCA simulations only include kinetic effects, so the discrepancies shown in Fig. 5 are to be expected. Similar to the results in the previous section, significant deviations between the simulated yields from SDTrimSP and SRIM-2013 exist, with the latter consistently predicting higher sputtering yields. Experimental yields for charge states higher than $1+$ are affected by potential sputtering and should therefore be higher than the simulation's predictions, which is not fulfilled for SRIM. Because of this and due to the excellent agreement for $2 \mathrm{keV} \mathrm{Ar}{ }^{+}$, SDTrimSP results were chosen as a reference for the kinetic contribution to the sputtering yield.

Ar occurs with energies between $20 \mathrm{keV}$ and $50 \mathrm{keV}$ and charge states of 7+ up to $10+$ in the solar wind (Barghouty et al., 2011), so measuring wollastonite sputtering for bombardment by $40 \mathrm{keV} \mathrm{Ar}^{8+}$ ions should be representative for solar wind conditions. 
Here a yield of about $60 \mathrm{amu} /$ ion was found, which is a doubling due to the potential sputtering effects. As can be seen in Fig. 5, the experimental sputtering yield for $\mathrm{Ar}^{8+}$ is twice as high as the SDTrimSP prediction, which only gives the kinetic sputtering yield.

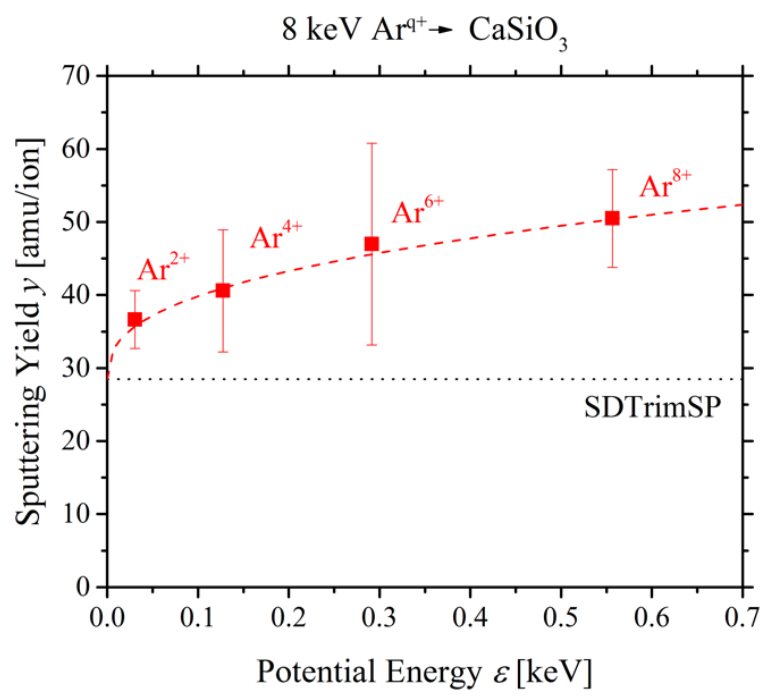

Fig. 6 Measured sputtering yields for different Ar charge states at a constant kinetic energy of $8 \mathrm{keV}$ under normal incidence.

At the constant kinetic impact energy of $8 \mathrm{keV}$, sputtering yields for the Ar charge states $2+, 4+, 6+$ and $8+$ were measured showing a clear increase of the yield for higher charge states. In Fig. 6, the respective experimental sputtering yields $y$ are plotted versus the ions' neutralization energy, i.e. the potential energy (with values taken from DREEBIT, 2018), which is responsible for the potential sputtering effects. The dotted black horizontal line indicates the SDTrimSP sputtering yield for $8 \mathrm{keV} \mathrm{Ar}^{+}$ions $(y=28.48$ amu/ion) and represents the kinetic sputtering contribution of a neutral projectile. After subtracting this contribution the remaining Ar potential sputtering yield can be well fitted using a fitting formula, which is based on work by Tona et al. and Ghose et al. (Ghose et al., 2003; Hijazi et al., 2014; Tona et al., 2005). The formula can be written as $\alpha \cdot \varepsilon^{\beta}$, where a least-square error fit results in $\alpha=26.88$ and $\beta=0.35$ (see dashed red line in Fig. 6) for the potential sputtering of wollastonite. Here the constant $\alpha$ is dependent on the material and $\varepsilon$ represents the ion's potential energy in keV minus twice the material's band gap (Hijazi et al., 2017), which is $5.4 \mathrm{eV}$ for wollastonite (Henriques et al., 2006). In this model, projectiles with potential energies below $10.8 \mathrm{eV}$ cannot cause potential sputtering, which is in accordance with the observed potential energy threshold at around 
$10 \mathrm{eV}$ for the potential sputtering of a LiF target (Aumayr and Winter, 2004). The formula has successfully been applied by Hijazi et al. to fit the potential sputtering contribution of anorthite (Hijazi et al., 2017) and we find that it also works very well for our wollastonite results. In the following it is therefore used to estimate the potential sputtering contribution for other solar wind relevant ions, since it can be assumed that the potential sputtering is to a large extent only dependent on the ion's potential energy (Aumayr and Winter, 2004).

\subsection{Angular Dependence under H Ion Irradiation}

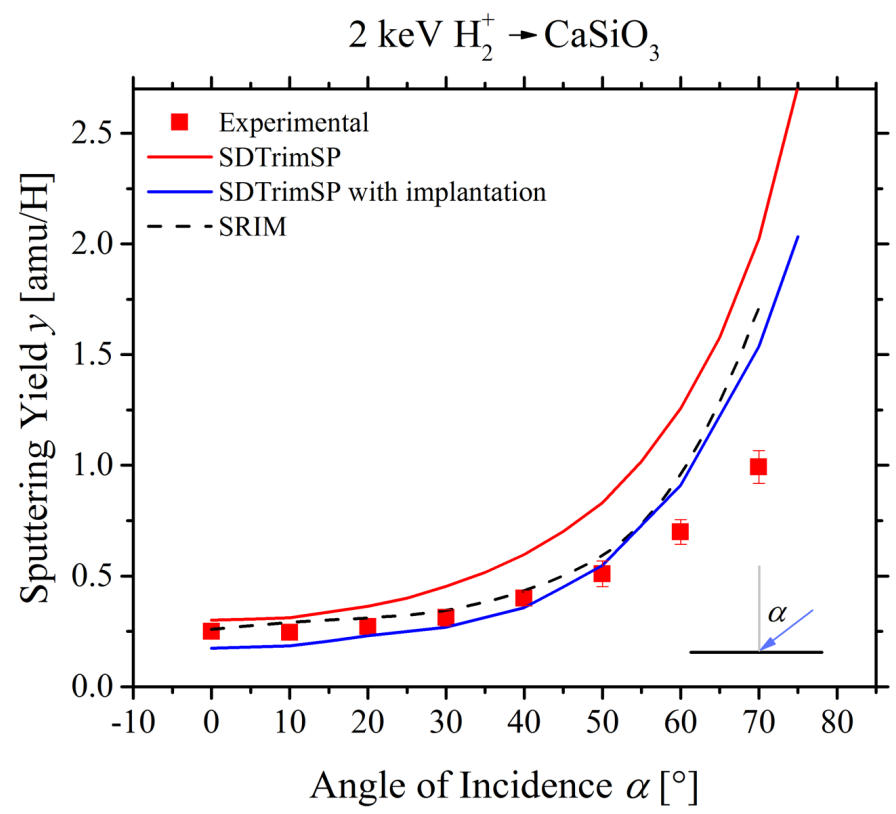

Fig. 7 Wollastonite sputtering yields under $\mathrm{H}_{2}{ }^{+}$irradiation, compared to SRIM and SDTrimSP simulations.

About $93 \%$ of the solar wind ions are made up by protons and therefore, sputtering of wollastonite by $\mathrm{H}_{2}{ }^{+}$projectiles was investigated for a kinetic energy of $2 \mathrm{keV}$. This represents an impact energy of $1 \mathrm{keV}$ per proton which is typical for solar wind protons. Results for the sputtering yield in $\mathrm{amu} / \mathrm{H}$ are shown in Fig. 7 as a function of ion impact angle and compared to the simulations. In contrast to $\mathrm{Ar}^{+}$bombardment, SDTrimSP only shows a good agreement for angles close to normal incidence, while the SRIM results fit our experimental data better. Still, sputter yield values for shallow angles of incidence are considerably over-estimated by both programs. 
Again we can assume that potential sputtering for protons can be neglected (see e.g. Potter, 1995). Chemical sputtering cannot be excluded completely, but it would increase the difference between experiment and simulation rather than explain it. One aspect, however, so far not included in the simulations is the implantation of $\mathrm{H}$ into the wollastonite sample. Contrary to Ar, $\mathrm{H}$ will form bonds with the target atoms and the $\mathrm{H}$ concentration inside the sample will rise. This in turn will alter the sputtering process since now also the implanted $\mathrm{H}$ atoms will be sputtered. SRIM and the static version of SDTrimSP are not able to account for this effect. The dynamic version of SDTrimSP allows simulating the change in sample composition due to the implantation of $\mathrm{H}$ atoms in the target. However, a complete description of the $\mathrm{H}$ release apart from sputtering is missing in the program. While SDTrimSP can theoretically simulate diffusion processes, experimental values for diffusion coefficients would be needed to allow the interpretation of the simulated results.

Nevertheless, a steady state-scenario where an equilibrium between $\mathrm{H}$ implantation and release exists can be reproduced by artificially restricting the maximum $\mathrm{H}$ content during such a dynamic simulation. Schaible and Baragiola showed that the initial probability of $\mathrm{OH}$ bond formations for $\mathrm{H}^{+}$bombardment of $\mathrm{SiO}_{2}$ and Olivine, which contains $\mathrm{Mg}_{2} \mathrm{SiO}_{4}$ and $\mathrm{Fe}_{2} \mathrm{SiO}_{4}$, is close to one (Schaible and Baragiola, 2014). With higher $\mathrm{H}$ fluences it then decreases exponentially as less $\mathrm{O}$ for $\mathrm{OH}$ formation becomes available. A reasonable maximal $\mathrm{H}$ concentration can thus be found, around the value where $\mathrm{H}$ and $\mathrm{O}$ concentrations are equal. By adding $\mathrm{H}$ to the sample composition from the XPS analysis (Table 1) and assuming that the relative abundances of the sample elements stay constant during the dynamic simulation, equal $\mathrm{H}$ and $\mathrm{O}$ parts are derived when both concentrations are at 35\%. A static SDTrimSP simulation, which includes the effect of $\mathrm{H}$ implantation by assuming a $\mathrm{H}$ concentration of $35 \%$ in the target, (blue line in Fig. 7) results in lower sputtering yields, leading to improved agreement for angles of around 50 degrees. Therefore, this represents a strong hint that the smaller experimental sputtering yields might be caused by the $\mathrm{H}$ concentration in the sample due to projectile implantation. 


\section{Modelling of Solar Wind Sputtering}

Taking into account all results from the previous sections, solar wind sputtering of wollastonite was modelled with SDTrimSP. Static calculations were performed under different incidence angles for the nominal $\mathrm{CaSiO}_{3}$ composition under irradiation of $\mathrm{H}, \mathrm{He}$, $\mathrm{C}, \mathrm{N}, \mathrm{O}, \mathrm{Ne}, \mathrm{Mg}, \mathrm{Si}, \mathrm{S}, \mathrm{Ar}$ and $\mathrm{Fe}$. Impact energies were chosen to match the slow solar wind velocity of $450 \mathrm{~km} / \mathrm{s}$, which corresponds to the typical kinetic energy per mass of 1 $\mathrm{keV} / \mathrm{amu}$ of the solar wind. For each species one medium charge state representative for the solar wind was assumed as specified in Table 2. The calculated kinetic sputtering yield values were adapted according to the experimental results. For $\mathrm{H}$, the simulated yields were rescaled to lower values so that they fit the experimental values, which leads to corrections especially for higher angles of incidence. For all projectiles apart from $\mathrm{H}$, potential sputtering contributions were added using the fit formula derived in Section 3.2. The potential sputtering was assumed to be independent of the different incidence angle. Furthermore, no implantation or chemical effects apart from the correction for $\mathrm{H}$ were taken into account.

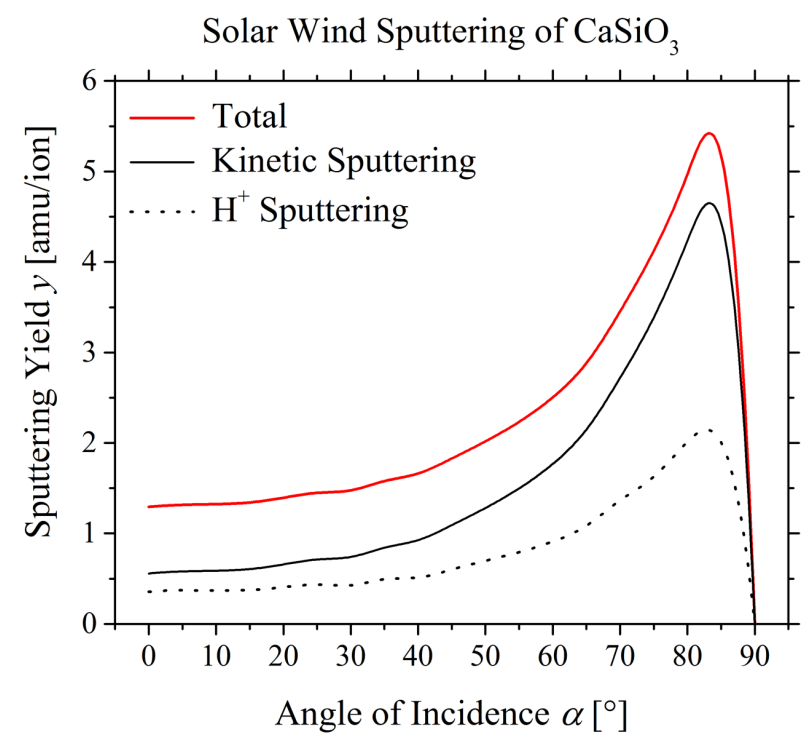

Fig. 8 Complete modelling of the wollastonite sputtering yield for an average solar wind ion, compared to kinetic and proton-only contributions. 


\begin{tabular}{|c|c|c|c|c|c|c|}
\hline Element & $\begin{array}{c}\text { Charge } \\
\text { State }\end{array}$ & $\begin{array}{c}\text { Potential } \\
\text { Energy [keV] }\end{array}$ & $\begin{array}{c}\text { Abundance } \\
\text { (relative to H) }\end{array}$ & $\begin{array}{c}\left.\boldsymbol{y} \mathbf{( 0}^{\circ}\right)- \\
\text { SDTrimSP } \\
\text { [amu/ion] }\end{array}$ & $\begin{array}{c}\boldsymbol{y}\left(\mathbf{0}^{\circ}\right)- \\
\text { corrected } \\
\text { [amu/ion] }\end{array}$ & $\begin{array}{c}\boldsymbol{y}\left(\mathbf{0}^{\circ}\right)- \\
\text { weighted } \\
\text { [amu/ion] }\end{array}$ \\
\hline $\mathrm{H}$ & $1+$ & 0.013 & 1 & 0.43 & 0.35 & 0.3310 \\
$\mathrm{He}$ & $2+$ & 0.077 & $7 \cdot 10^{-2}$ & 2.55 & 12.92 & 0.8435 \\
$\mathrm{C}$ & $5+$ & 0.536 & $5 \cdot 10^{-4}$ & 11.86 & 33.35 & 0.0156 \\
$\mathrm{~N}$ & $6+$ & 0.814 & $5 \cdot 10^{-5}$ & 14.30 & 39.22 & 0.0018 \\
$\mathrm{O}$ & $6+$ & 0.428 & $7 \cdot 10^{-4}$ & 16.75 & 36.59 & 0.0239 \\
$\mathrm{Ne}$ & $8+$ & 0.945 & $9 \cdot 10^{-5}$ & 21.20 & 47.45 & 0.0040 \\
$\mathrm{Mg}$ & $8+$ & 1.026 & $1 \cdot 10^{-4}$ & 25.92 & 52.95 & 0.0049 \\
$\mathrm{Si}$ & $9+$ & 1.369 & $1 \cdot 10^{-4}$ & 30.39 & 60.29 & 0.0056 \\
$\mathrm{~S}$ & $8+$ & 0.878 & $3 \cdot 10^{-5}$ & 34.51 & 60.10 & 0.0017 \\
$\mathrm{Ar}$ & $8+$ & 0.567 & $4 \cdot 10^{-6}$ & 39.68 & 60.23 & 0.0002 \\
$\mathrm{Fe}$ & $10+$ & 1.041 & $8 \cdot 10^{-4}$ & 54.00 & 81.16 & 0.0605 \\
\hline & & & & Total: & 1.2928 \\
\hline
\end{tabular}

Table 2 Overview of the ions included in the model for solar wind sputtering of wollastonite.

All the SDTrimSP results for the different projectiles were weighted according to the relative solar wind abundances of the respective elements (taken from (Bame et al., 1975; Barghouty et al., 2011; Russell et al., 2016)) and added to derive a mean solar wind sputtering yield for wollastonite. Fig. 8 shows the resulting angular dependence of the sputtering yield where the average yield per solar wind ion (red line) is plotted together with the kinetic contribution (black line) and the $\mathrm{H}^{+}$contribution (dotted line). Table 2 gives a detailed overview of the different projectiles that were used. The potential energies that are given in the third column were taken from (DREEBIT, 2018).

A very prominent angular dependence can be seen in Fig. 8, with the average sputtering yield increasing from $1.3 \mathrm{amu} /$ ion for normal incidence to about $5.3 \mathrm{amu} /$ ion for $80^{\circ}$ incidence. As discussed in Section 2.3, this will strongly influence the sputtering yields of rough samples and is thus highly relevant in a realistic environment. Potential sputtering cannot be neglected either, since it doubles the total yield at normal incidence, while it becomes less important for large angles of incidence. This is a consequence of our 
assumption that the potential sputtering contribution is largely independent on the incidence angle, a fact which needs to be experimentally verified in the future.

Looking at Table 2, about two thirds of the sputtering yield is caused by He projectiles. $\mathrm{He}^{2+}$ already shows a significant potential sputtering contribution and has a relatively high concentration in the solar wind compared to heavier ions. Besides $\mathrm{H}$ and $\mathrm{He}$, the multiply charged $\mathrm{Fe}, \mathrm{O}$ and $\mathrm{C}$ ions still contribute between about $1 \%$ and $5 \%$ to the total solar wind sputtering yield, while the contributions from other elements are mostly negligible in this calculation.

Compared to proton sputtering at normal incidence, kinetic sputtering by heavier ions increases the yield by $56 \%$ and the inclusion of potential sputtering by $260 \%$. These factors are higher than proposed by Barghouty et al. for Lunar KREEP material who reported increases by 26\% and 52\% (Barghouty et al., 2011) and by Hijazi et al. for Anorthite $\mathrm{CaAl}_{2} \mathrm{Si}_{2} \mathrm{O}_{8}$ of $25 \%$ and $50 \%$, (Hijazi et al., 2017). As mentioned before, a substantial part of the sputtering yield in our model is caused by $\mathrm{He}^{2+}$, where the predicted total sputtering yield (including the potential contribution) is five times higher than the kinetic one. This dominance of $\mathrm{He}$ is in agreement with previous findings for solar wind sputtering (Hijazi et al., 2017). The exact results are however very sensitive to the potential sputtering model used, which is why experiments with $\mathrm{He}^{2+}$ projectiles should be carried out in the future to validate the potential sputtering formula.

At the maximum of the yield's angular dependence at an angle of incidence between 80 and 85 degrees, the role of heavy ions is also important. Kinetic sputtering is increased by $117 \%$, while the inclusion of potential sputtering increases the yield by $153 \%$ compared to proton sputtering. This dominance of heavier ions at large angles is not predicted by SDTrimSP, but a consequence of the experimentally observed less pronounced angular dependence of the proton sputtering yield, which may be caused by $\mathrm{H}$ implantation in wollastonite. 


\section{Conclusion and Outlook}

Sputtering experiments with the well-established QCM method were conducted using laser-deposited wollastonite targets under bombardment by $\mathrm{Ar}$ and $\mathrm{H}$ ions at various impact angles. Theoretical values were calculated with the BCA simulations SDTrimSP and TRIM to compare how well the commonly used simulation programs are able to describe the sputtering yields. For $\mathrm{Ar}^{+}$bombardment, which represents a case where the sputtering should only be of kinetic nature, SDTrimSP gives a very precise result for the sputtering yield's angular dependence. Here SDTrimSP represents a substantial improvement over SRIM-2013. For higher Ar charge states potential sputtering effects increase the sputtering yield to up to twice the value of kinetic sputtering. The influence of the potential energy on this increase was determined by a fit to experimental data using a common model. Hydrogen bombardment showed much smaller sputtering yields than Ar with $\mathrm{H}$ implantation probably representing an important aspect of the sputtering process.

Based on these experimental results and extrapolation to other solar wind ions by using SDTrimSP, a general simulation of solar wind induced sputtering of wollastonite was performed. A very prominent dependence on the ion impact angle was found with potential sputtering having a substantial contribution at normal incidence. These investigations confirm that heavier, multiply charged ions play a significant role in the solar wind sputtering, in particular $\mathrm{He}^{2+}$. Therefore, this model would benefit from obtaining experimental results for wollastonite sputtering by $\mathrm{He}^{2+}$.

Another aspect that remains to be further investigated is the $\mathrm{H}$ implantation. An adapted SDTrimSP simulation led to an improved description, but the detailed explanation of the sputtering yield for higher angles of incidence is still lacking. Maybe the trapping of $\mathrm{H}_{2}$ gas bubbles inside the wollastonite target as well as chemical sputtering effects need to be considered. Chemical sputtering has been extensively investigated for the $\mathrm{H}$ bombardment of C-containing samples (Hopf et al., 2003). The chemical sputtering yield has been found to be dependent on the ion's impact energy, but also on the ion flux and the sample-temperature (Jacob and Roth, 2007). On planets like Mercury, surface temperatures of several hundred ${ }^{\circ} \mathrm{C}$ can be expected where such chemical processes would be accelerated and become even more prominent. Further investigations at higher 
temperatures, lower impact energies and different ion fluxes would then give insight into the role of chemical sputtering for wollastonite.

In a realistic environment, solar wind sputtering will not occur on flat samples, but on rough rocks or small grains. The roughness and exact shape of the sample will greatly influence the sputtering yields due to a range of different local angles of incidence, redeposition of sputtered material, secondary sputtering by reflected projectiles and shadowing effects. QCM targets are limited in this aspect because they only allow the investigation of thin films. Therefore, using the catcher-QCM setup at TU Wien that allows to collect the sputtered material from a more realistic target (Berger et al., 2017), as well as using the enhanced versions SDTrimSP-2D and SDTrimSP-3D (Mutzke et al., 2013; von Toussaint et al., 2017) will add more insight into the influence of surface roughness for solar wind sputtering. 


\section{Acknowledgments}

The authors are grateful to Michael Schmid (IAP, TU Wien) for his continued support with the QCM electronics. Financial support has been provided by KKKÖ (commission for the coordination of fusion research in Austria at the Austrian Academy of Sciences - ÖAW). 


\section{References}

Aumayr, F., Winter, H., 2004. Potential sputtering. Philosophical Transactions of the Royal Society of London A: Mathematical, Physical and Engineering Sciences. 362, 77-102.

Bame, S. J., Asbridge, J. R., Feldman, W. C., Montgomery, M. D., Kearney, P. D., 1975. Solar wind heavy ion abundances. Solar Physics. 43, 463-473.

Barghouty, A. F., Meyer, F. W., Harris, P. R., Adams, J. H., 2011. Solar-wind protons and heavy ions sputtering of lunar surface materials. Nuclear Instruments and Methods in Physics Research Section B: Beam Interactions with Materials and Atoms. 269, 1310-1315.

Berger, B. M., Szabo, P. S., Stadlmayr, R., Aumayr, F., 2017. Sputtering measurements using a quartz crystal microbalance as a catcher. Nuclear Instruments and Methods in Physics Research Section B: Beam Interactions with Materials and Atoms. 406, 533-537.

Dobes, K., Naderer, P., Hopf, C., Schwarz-Selinger, T., Aumayr, F., 2012. Transient effects during sputtering of a-C:H surfaces by nitrogen ions. Nuclear Instruments and Methods in Physics Research Section B: Beam Interactions with Materials and Atoms. 286, 20-24.

DREEBIT - Ionization Energy Database, http://www.dreebit-ibt.com/ionizationenergy-database.html

Galutschek, E., Trassl, R., Salzborn, E., Aumayr, F., Winter, H. P., 2007. Compact 14.5 $\mathrm{GHz}$ all-permanent magnet ECRIS for experiments with slow multicharged ions. Journal of Physics: Conference Series, Vol. 58. IOP Publishing, pp. 395.

Ghose, D., Karmakar, P., Parilis, E., 2003. Evidence of Coulomb explosion sputtering of ultra-thin $\mathrm{Pt}$ films due to impact of multi-charged Ar ions. Nuclear Instruments and Methods in Physics Research Section B: Beam Interactions with Materials and Atoms. 212, 420-425.

Golczewski, A., Dobes, K., Wachter, G., Schmid, M., Aumayr, F., 2009. A quartzcrystal-microbalance technique to investigate ion-induced erosion of fusion relevant surfaces. Nuclear Instruments and Methods in Physics Research Section B: Beam Interactions with Materials and Atoms. 267, 695-699.

Grün, E., Horanyi, M., Sternovsky, Z., 2011. The lunar dust environment. Planetary and Space Science. 59, 1672-1680.

Hapke, B., 1986. On the sputter alteration of regoliths of outer solar system bodies. Icarus. 66, 270-279.

Hapke, B., 2001. Space weathering from Mercury to the asteroid belt. Journal of Geophysical Research: Planets. 106, 10039-10073.

Hayderer, G., et al., 2001. Kinetically assisted potential sputtering of insulators by highly charged ions. Physical Review letters. 86, 3530-3533.

Hayderer, G., Schmid, M., Varga, P., Winter, H. P., Aumayr, F., 1999. A highly sensitive quartz-crystal microbalance for sputtering investigations in slow ionsurface collisions. Review of Scientific Instruments. 70, 3696.

Henriques, J., Caetano, E., Freire, V., Da Costa, J., Albuquerque, E., 2006. Structural and electronic properties of $\mathrm{CaSiO} 3$ triclinic. Chemical Physics Letters. 427, 113-116.

Hijazi, H., Bannister, M., Meyer, H., Rouleau, C. M., Meyer, F., 2017. Kinetic and potential sputtering of an anorthite-like glassy thin film. Journal of Geophysical Research: Planets. 122, 1597-1609.

Hijazi, H., et al., 2014. Anorthite sputtering by $\mathrm{H}+$ and $\mathrm{Arq}+(\mathrm{q}=1-9)$ at solar wind velocities. Journal of Geophysical Research: Space Physics. 119, 8006-8016. 
Hofsäss, H., Zhang, K., Mutzke, A., 2014. Simulation of ion beam sputtering with SDTrimSP, TRIDYN and SRIM. Applied Surface Science. 310, 134-141.

Hopf, C., Von Keudell, A., Jacob, W., 2003. Chemical sputtering of hydrocarbon films. Journal of Applied Physics. 94, 2373-2380.

Jacob, W., Roth, J., 2007 Chemical sputtering. Sputtering by Particle Bombardment (R. Behrisch, W. Eckstein). Springer, pp. 329-400.

Kracher, A., Aumayr, F., Sears, D., Kareev, M., 2003. Space weathering by highly charged heavy ions in the solar wind. Meteoritics and Planetary Science Supplement. 38.

Küstner, M., Eckstein, W., Dose, V., Roth, J., 1998. The influence of surface roughness on the angular dependence of the sputter yield. Nuclear Instruments and Methods in Physics Research Section B: Beam Interactions with Materials and Atoms. 145, 320-331.

Küstner, M., Eckstein, W., Hechtl, E., Roth, J., 1999. Angular dependence of the sputtering yield of rough beryllium surfaces. Journal of Nuclear Materials. 265, 22-27.

Loeffler, M. J., Dukes, C. A., Baragiola, R. A., 2009. Irradiation of olivine by 4 keV He+: Simulation of space weathering by the solar wind. Journal of Geophysical Research: Planets. 114.

Mutzke, A., Eckstein, A., Schneider, R., Dohmen, R., 2011. SDTrimSP: Version 5.00. IPP Report $12 / 8$.

Mutzke, A., Schneider, R., Bandelow, G., 2013. SDTrimSP-2D: Simulation of Particles Bombarding on a Two Dimensional Target-Version 2.0. IPP Report 12/11.

Orsini, S., et al., 2010. SERENA: A suite of four instruments (ELENA, STROFIO, PICAM and MIPA) on board BepiColombo-MPO for particle detection in the Hermean environment. Planetary and Space Science. 58, 166-181.

Pfleger, M., et al., 2015. 3D-modeling of Mercury's solar wind sputtered surfaceexosphere environment. Planetary and Space Science. 115, 90-101.

Pieters, C. M., Noble, S. K., 2016. Space weathering on airless bodies. Journal of Geophysical Research: Planets. 121, 1865-1884.

Potter, A., 1995. Chemical sputtering could produce sodium vapor and ice on Mercury. Geophysical research letters. 22, 3289-3292.

Russell, C. T., Luhmann, J. G., Strangeway, R. J., 2016. Space Physics: An Introduction. Cambridge University Press.

Sauerbrey, G., 1959. Verwendung von Schwingquarzen zur Wägung dünner Schichten und zur Mikrowägung. Zeitschrift für Physik. 155, 206-222.

Schaible, M. J., Baragiola, R. A., 2014. Hydrogen implantation in silicates: the role of solar wind in $\mathrm{SiOH}$ bond formation on the surfaces of airless bodies in space. Journal of Geophysical Research: Planets. 119, 2017-2028.

Tona, M., et al., 2005. Coulomb explosion potential sputtering induced by slow highly charged ion impact. Applied Physics Letters. 87, 224102.

Vervack, R. J., et al., 2010. Mercury's complex exosphere: Results from MESSENGER's third flyby. Science. 329, 672-675.

von Toussaint, U., Mutzke, A., Manhard, A., 2017. Sputtering of rough surfaces: a 3D simulation study. Physica Scripta. Volume 2017, 014056.

Wurz, P., 2005. Solarwind composition. The Dynamic Sun: Challenges for Theory and Observations, Vol. 600.

Wurz, P., et al., 2007. The lunar exosphere: The sputtering contribution. Icarus. 191, 486-496.

Wurz, P., Whitby, J. A., Rohner, U., Martín-Fernández, J. A., Lammer, H., Kolb, C., 2010. Self-consistent modelling of Mercury's exosphere by sputtering, micro- 
meteorite impact and photon-stimulated desorption. Planetary and Space Science. 58, 1599-1616.

Ziegler, J. F., Ziegler, M. D., Biersack, J. P., 2010. SRIM-The stopping and range of ions in matter (2010). Nuclear Instruments and Methods in Physics Research Section B: Beam Interactions with Materials and Atoms. 268, 1818-1823. 